\title{
Evaluation of soluble TNF-like weak inducer of apoptosis (sTWEAK) levels to predict preeclampsia in early weeks of pregnancy
}

\author{
Sana Shahid \\ Sir Syed College of Medical Sciences for Girls, Karachi, Pakistan \\ Erum Khalid \\ Taj Medical Complex, Hamdard University, Pakistan \\ Syeda Sadia Fatima \\ Aga Khan University, sadia.fatima@aku.edu \\ Ghulam Mustafa Khan \\ Basic Medical Science Institute, Karachi, Pakistan
}

Follow this and additional works at: https://ecommons.aku.edu/pakistan_fhs_mc_bbs

Part of the Biochemistry, Biophysics, and Structural Biology Commons

\section{Recommended Citation}

Shahid, S., Khalid, E., Fatima, S. S., Khan, G. M. (2019). Evaluation of soluble TNF-like weak inducer of apoptosis (sTWEAK) levels to predict preeclampsia in early weeks of pregnancy. European Journal of Obstetrics and Gynecology and Reproductive Biology, 234, 165-170.

Available at: https://ecommons.aku.edu/pakistan_fhs_mc_bbs/740 
Title

2
Evaluation of Soluble TNF-like weak inducer of apoptosis (sTWEAK) Levels to Predict Preeclampsia in Early Weeks of Pregnancy

Short title

sTWEAK in Preeclampsia Sana Shahid ${ }^{1}$, Erum Khalid ${ }^{2}$, Syeda Sadia Fatima ${ }^{3 *}$, Ghulam Mustafa Khan ${ }^{4}$

${ }^{1}$ Department of Physiology, Sir Syed College of Medical Sciences for Girls, Karachi, Pakistan, ${ }^{2}$ Department of Obstetrics and Gynecology, Taj Medical Complex, Hamdard University, ${ }^{3}$ Department of Biological and Biomedical Sciences, Aga Khan University, Karachi, Pakistan, ${ }^{4}$ Department of Physiology, Basic Medical Science Institute, Karachi, Pakistan

\section{Corresponding Author:}

Syeda Sadia Fatima

Department of Biological and Biomedical Sciences, Aga Khan University, Karachi, Pakistan,

Phone: +92-21-34864564

E-mail: sadia.fatima@aku.edu

Disclosure statement: All authors declare that they have no potential conflict of interest

Funding: Pakistan Health Research Counsel (Ref No. 119/2016/RDC/AKU). Awarded to SSF

\section{Author Contribution:}

SSF conceived the project, analyzed the data and wrote the paper. SS collected and followed study subject, performed the experiments and wrote the paper. EK and GMK wrote the paper. All authors approved the final version before submission and publication. 
32 Introduction: Soluble tumor necrosis factor-like weak inducer of apoptosis 33 (sTWEAK) is linked to endothelial dysfunction; a key factor in pre-eclamptic 34 pathogenesis. This study aimed to compare sTWEAK levels during pregnancy to 35 assess for its prognostic ability.

36 Materials and Methods: Sixty three high risk pregnant women were followed up from 3712 weeks of gestation till term. Serum levels of sTWEAK and platelet derived growth 38 factor (PlGF), blood pressure, serum glucose, uric acid, urea/creatinine and liver 39 function tests were measured. Subjects were stratified according to the ACOG criteria 40 as women who developed $\mathrm{PE}$, or $\mathrm{PIH}$ or remained normotensive at term. A negative 41 control group of normotensive healthy pregnant women $(n=17)$ was also recruited for 42 comparison.

43 Results: Baseline sTWEAK levels were lower $(4.03 \pm 0.37 \mathrm{ng} / \mathrm{dl})$ in $\mathrm{HR}$ cohort that 44 developed PE and further reduced at term $(1.93 \pm 0.23 \mathrm{ng} / \mathrm{dl})$ as compared to HR subjects 45 who remained normotensive and negative control group $(30.53 \pm 0.79 \mathrm{ng} / \mathrm{dl} ; \mathrm{p}<0.01)$. 46 Likewise PlGF levels were significantly lower $(74.22 \pm 10.11 \mathrm{pg} / \mathrm{ml})$ in $\mathrm{HR}$ cohort that 47 developed PE $(\mathrm{p}=0.013)$. At term 39.68\% $(\mathrm{n}=22)$ HR subjects with low sTWEAK 48 developed PIH and 34.92\% ( $\mathrm{n}=24)$ developed PE. In terms of high risk characteristics 49 observed in the HR group; $73 \%$ of the subjects were multiparous, whereas $26.98 \%$ 50 reported to have developed PE in previous pregnancies.

51 Conclusion: sTWEAK levels at early pregnancy weeks were found to be low in high 52 risk females who developed PE at follow up versus normotensive pregnant women. 53 Baseline TWEAK might serve as an independent variable for prediction of pre54 eclampsia; however longitudinal studies with larger sample size are required to 55 ascertain the causal relation.

56 Key words: Hypertension; Pregnancy; Pre-eclampsia; sTWEAK 


\section{Introduction}

59 Pre-eclampsia, is characterized by sudden onset of hypertension and proteinuria in 60 women with no preceding hypertensive history. Due to its dormant nature, it possess 61 great threat to maternal as well as fetal wellbeing (1). Globally 5-14\% of all 62 pregnancies are complicated by $\mathrm{PE}$ and in developing countries it's prevalence is 4$6318 \%$ (2). $\mathrm{PE}$ is a multifactor disorder, starting with placental dysfunction leading to 64 augmented anti-angiogenic response in mother (3). With the loss of balance between 65 pro- and antiangiogenic factors, the maternal endothelial function deteriorates (4). It 66 has also been proposed that normal pregnancy features subservient inflammatory 67 response in itself, however, in PE this response is augmented. The augmented systemic 68 response causes dysfunction in maternal endothelium by involving maternal 69 leukocytes, platelets and also activating pro-inflammatory cytokines (5-7).

70 For PE, early prediction has always been the primary priority of many clinician and 71 researchers. Previous maternal history and presence of risk factors alone are not 72 reliable for the prediction; therefore more profound monitoring is required for prompt 73 diagnosis and fruitful treatment strategies. Keeping this in view, detection of PE 74 biomarkers in early pregnancy has always been an interesting field of research (8). 75 Placental growth factor (PlGF), pregnancy associated plasma protein A (PAPP-A), 76 Free fetal hemoglobin (HbF), Soluble Endoglin and placental protein 13 (PP-13) are 77 among some of the commonly studied biomarkers for PE pathology (9, 10) (11). Yet 78 the predictive value as a single most important, reliable, and effective marker has not 79 been proven till date (12). Among previously identified predictive serum proangiogenic 80 biomarker related to pre-eclamptic pathogenesis is placental growth factor (PlGF) (13). 81 PlGF is a member of vascular endothelial growth factor (VEGF) family and is highly 82 expressed in placental tissue for healthy placentation (14). The placental angiogenic 83 factors are responsible for proper placental development through neovascularization, 84 cellular remolding and maintaining nitric oxide levels. Lack of these factors seem to 85 play role in defective placentation (15). Serum PIGF levels were found to be reduced in 86 early weeks of pregnancy by multiple studies $(16,17)$, thus indicating its role in pre87 eclamptic pathogenesis. 
Recently, a new serum marker, soluble tumor necrosis factor like weak inducer of apoptosis (sTWEAK), has been proposed to be altered in the maternal blood. sTWEAK is a multipurpose cytokine which is involved in conducting diverse biological events,

91 like cellular multiplication, growth, migration, angiogenesis, cell differentiation, 92 apoptosis and inflammation by instigating expression of multiple pro-inflammatory 93 cytokines. Furthermore, in peculiar it appears to perform critical role in tissue repair 94 and wound healing (3). TWEAK is universally expressed as Type II trans-membrane 95 protein of 35-kDA which after cleaving, shed functionally active soluble 18-kDA processed factor called as STWEAK (18). sTWEAK is widely expressed in many different tissues (19). Overall data suggests that sTWEAK may have physiological as well as pathological responses in tissues. On one hand it is known to induce proliferation of endothelial cells in vitro and angiogenesis in vivo and on other hand triggers the production of pro-inflammatory cytokines (20). Binding of sTWEAK to its receptor results in activation of any one of the three effector pathways; a) proliferative, b) inflammatory or c) apoptotic, in which the inflammatory pathway being the most dominant one. The other 2 pathway activation depends on the cellular integrity. With intact cellular physiological mechanisms along with absence of inflammatory process, the effector pathway will be the proliferative pathway, however in the presence of proinflammatory cytokines (TNF- $\alpha$, IFN $\gamma$ ), the predominant pathway is the apoptotic one (21). In this context, Donohue and group have demonstrated the in vivo stimulatory effect of sTWEAK on angiogenesis in human endothelial cells $(3,22)$.

109 Since pregnancy is the condition comprising of both angiogenesis and mild 110 inflammation; there might be possibility of sTWEAK's involvement in these processes 111 at the time of placentation. Further any alteration in the levels of sTWEAK and PlGF 112 in the beginning of pregnancy may lead to development of PE. Keeping the literature in 113 view, we proposed that there might be close relation of pre-eclamptic pathogenesis to 114 disrupted angiogenic system and endothelial dysfunction caused by low sTWEAK. 115 Hence, this study aimed to compare sTWEAK levels during pregnancy to assess for its 116 prognostic ability. 


\section{Methods:}

119 This prospective study was conducted from January 2017 to March 2018. The study was 120 approved by the Intuitional Review Board (IRB) of Jinnah Post Graduate Medical Centre 121 (JPMC) (Ref: NO.F.2-81/GENL-2017-IRB/15107/JPMC) and Aga Khan University (4523-BBS122 ERC-16) in collaboration with Taj Medical Complex, Karachi. The minimum sample required 123 for this study was 60 subjects with a 95\% confidence interval and a $4 \%$ frequency of outcome 124 factor in the population (23). A total of $n=137$ pregnant women were initially enrolled from the 125 obstetrical clinics of these hospitals; out of these $n=80$ subjects were successfully followed till the end of study and were included in this manuscript.

127 Out of the study cohort; 63 subjects were classified as high risk pregnant women (HR group).

128 The HR status was based on the presence of any of the criteria: first pregnancy/ family history of $129 \mathrm{PE} /$ multiple gestation/ previous history of PE/ maternal age up to 35 years / BMI of $>35 \mathrm{~kg} / \mathrm{m}^{2}$ 130 and or presence of chronic hypertension. These subjects were followed up from 12 weeks of gestation till term (observed for development of PE/HTN). Pre-eclampsia diagnoses was based on the American College of Obstetricians and Gynecologists (ACOG) criteria as follows:- values of systolic blood pressure $140 \mathrm{~mm} / \mathrm{Hg}$ or higher and diastolic blood pressure $90 \mathrm{~mm} / \mathrm{Hg}$ or higher after 20 weeks of gestation with or without dipstick proteinuria $\left(0.3 \mathrm{gm} / 1\right.$ or $\left.>1^{+}\right)$were taken as a reference (24). The remaining $(n=17)$ females who did not show any proteinuria or blood pressure derangements were labeled as pregnant negative controls. For all study subjects females with chronic systemic disease (cardiovascular, urogenital, immunological, endocrinological), renal disease, previous history of complication of pregnancy such as abortion, intra-uterine fetal demise, antenatal bleeding were excluded from the study.

An informed written consent form was signed by each subject. Their demographic 141 data, medical and obstetrical history and examination were recorded at the time of 142 enrolment on a predesigned form. Serum samples were obtained and analyzed for 143 complete blood count, random blood glucose, uric acid, urea/creatinine and liver 144 function test. Freshly voided early morning mid-stream urine sample was obtained for 145 estimation of proteinuria. The serum sTWEAK concentration was determined by using 146 Human sTWEAK ELISA Kit (Cat. No. H1911 by Glory Science Co, Ltd Belgium) and 147 PIGF (kit cat \#DPG00 by R\&D systems USA) according to the provided protocol. In 
148 high risk and control group, blood samples were collected a) at baseline (12-16weeks) 149 and b) follow-up (28-36 weeks gestation).

150 Statistical analysis was conducted by SPSS version 23.0. A descriptive statistical 151 analysis of continuous variables was performed. Data on continuous variables i.e. 152 biophysical (age, height, weight, blood pressure etc.) and biochemical (Serum 153 sTWEAK, PIGF, blood glucose, serum uric acid, serum creatinine, etc.) parameters 154 were expressed as Mean \pm standard deviation (SD) or standard error of mean (SEM) 155 whereas data on categorical variables were presented as absolute number and 156 percentages. Statistical comparisons were performed by using student t-test, paired 157 sample t-test and Mann Whitney-U-test for continuous/quantitative variables, chi158 square or Fisher exact test for categorical variables. In all statistical analysis only p159 value $<0.05$ was taken as significant.

\section{Results}

161 The detailed results of this study are shown in Tables 1-3. Table 1 shows the 162 demographic distribution of study cohort. Mean age, BMI, weight, hemoglobin levels 163 and blood glucose parameters were matched for each group therefore no difference was 164 seen $(\mathrm{p}>0.05)$. Alkaline phosphatase levels were slightly raised in HR (243.38 \pm 78.36 $165 \mathrm{mg} / \mathrm{dl})$ as compared to control group $(167.0 \pm 120.22 \mathrm{mg} / \mathrm{dl} ; \mathrm{p}=0.039)$. Serum uric acid 166 and urea showed no difference among the groups ( $p>0.05)$. In terms of high risk 167 characteristics observed in the HR group; 73\% study subjects were multiparous, 168 whereas $26.98 \%$ reported to have developed PE in previous pregnancies.

169 Table 2 shows the systolic and diastolic blood pressure of study subjects stratified 170 according to the HR outcome. Baseline blood pressure reading of each subject was 171 within normal range; whereas subjects who developed PIH $(n=24)$ or PE ( $n=22)$ had 172 significantly higher blood pressure readings than normotensive HR subjects ( $\mathrm{n}=17)$ and 173 negative control group $(\mathrm{n}=17)(\mathrm{p}<0.01)$. The urine dipstick assay for urine protein and 174 glucose showed $22 \%$ of HR subjects with positive proteinuria while $3.2 \%$ were 175 positive for glycosuria varying degrees. 
176 The assessment of baseline sTWEAK levels revealed a lower value $(4.03 \pm 0.37 \mathrm{ng} / \mathrm{dl})$ in 177 subjects that developed $\mathrm{PE}$ and were further reduced at term $(1.93 \pm 0.23 \mathrm{ng} / \mathrm{dl})$ in 178 comparison to normotensive HR subjects and negative control group $(\mathrm{p}<0.001)$. 179 Similar trend was observed for baseline and follow up sTWEAK levels of HR subjects 180 who developed PIH $(\mathrm{p}<0.001)$. Likewise, PIGF levels were significantly low in HR 181 cohort that developed PE $(74.22 \pm 10.11 \mathrm{ng} / \mathrm{dl})$ or PIH $(89.38 \pm 8.38 \mathrm{ng} / \mathrm{dl})$ as compared to 182 normotensive HR $(101.0 \pm 12.13 \mathrm{ng} / \mathrm{dl})$ and negative controls $(109.82 \pm 7.83 \mathrm{ng} / \mathrm{dl})$ 183 ( $\mathrm{p}=0.013)$ (Table 3). The pregnancy outcomes of these HR subjects were as follows: 2 184 IUGR; 3 IUD's; 19 LSCS; where the remaining 24 were delivered via simple vaginal 185 deliveries. 


\section{Discussion:}

188 Recent advancements in pre-eclamptic management has upgraded the level of safe 189 pregnancy and to some extent reduced the mortality and morbidity in developed 190 countries, however in developing countries there is still a need to improve the 191 pregnancy outcome by early detection of pregnancy complications. In this study, 192 sTWEAK has emerged as a promising contemporary biomarker for early pre-eclamptic 193 prediction in women having risk factors such as multiple pregnancies, previous history 194 of PE and age of up to 35 year. The study reports that a considerable number of 195 pregnant subjects with remarkably lower sTWEAK levels at baseline and follow up 196 developed either pre-eclampsia or pregnancy induced hypertension near term. This 197 finding reinforces the assumption that low sTWEAK is related with the progression or 198 disease severity (21). Therefore, it is plausible that this finding may give a novel 199 insight into the ability of STWEAK to identify high risk subjects during early 200 pregnancy weeks for the first time that may develop PE later. This finding is in 201 consensus with the only available published study which found decreased serum 202 sTWEAK concentration in pre-eclamptic women as compared to controls (p-value 203 0.04) (3). However, there is a slight difference in reported concentrations that may be 204 attributed to a different population, different time of sample collection and difference 205 assay protocol. Moreover, in this study sTWEAK levels were slightly lower (but not 206 below 9ng/dl) in normotensive HR subjects in comparison to pregnant controls, which 207 could be due to the presence of risk factors influencing the general condition of the 208 patients. In addition, these subjects had a normal PlGF level that might have 209 compensated for the changes in pregnancy.

210 Currently, no research data is available regarding the role of sTWEAK in pre-eclamptic 211 pathogenesis. However, several previous studies have identified sTWEAK as a potent 212 inducer of angiogenesis, acting as a mitogenic factor for human endothelial cells (25).

213 It is also found to be linked with endothelial dysfunction in non-dialysis chronic kidney 214 disease, diabetic and renal transplant patients (26). Decreased sTWEAK concentrations 215 were also detected in conditions like atherosclerosis, coronary artery disease and 216 peripheral arterial disease (27). Another group linked lower sTWEAK to inflammatory 
217 changes in gestational diabetes (GDM) and insulin resistance (28); however in this 218 study only 2 cases of GDM were observed.

219 The current study also reports a reduced maternal serum PlGF level along with reduced 220 sTWEAK levels in early pregnancy indicating its link to pre-eclamptic pathogenesis. 221 Maternal PlGF involvement in proper placentation through effective angiogenesis has 222 been proved previously (29). PlGF works as a mitogenic factor for endothelial cells 223 and its levels are high throughout the pregnancy (14), therefore, the reduced levels of 224 PlGF may predict pre-eclampsia (30). However, studies have proposed that PlGF alone 225 has a limited predictive capacity (31). The predictive value of most of the biomarkers 226 working as a single entity is not satisfactory unless a combination of markers are used 227 (9). Since this study suggests sTWEAK ability to predict PE as an independent marker, 228 introducing the new combination of sTWEAK and PIGF may enhance the screening 229 capabilities for the disease in early weeks of pregnancy.

230 The connection of sTWEAK with the pre-elcamptic pathogenesis can be explained by 231 its behavior as an inflammatory cytokine. Since pregnancy is considered as a low grade 232 inflammatory condition (32); sTWEAK physiological or pathological nature, may have 233 some contribution in pre-eclamptic pathogenesis, owing to the fact of exaggerated 234 maternal inflammatory response in pre-eclamptic pregnancy (33). Published data also 235 suggest the role of sTWEAK in neovascularization (25), there might be a possibility 236 that deficiency of sTWEAK in the blood is responsible for defective angiogenesis in 237 placenta leading to $\mathrm{PE}$ as seen in this study.

238 In Pakistan, the overall estimated incidence of intra uterine deaths is around 5.22\% (34) 239 which is linked to cases of antepartum hemorrhage, hypertensive disorders of 240 pregnancy (preeclampsia and eclampsia), mismanagement of labor and diabetes etc. In 241 this study, the number of IUD's reported was $3(4.76 \%)$ and IUGR was $2(3.17 \%)$ in 242 the PE/PIH group, which is comparable to the available data $(35,36)$.

243 This study was however limited on commenting on the prediction of early onset and 244 late onset PE as the number of patients in this study design was limited. Additionally, it 245 is uncertain whether sTWEAK is superior in predicting PE alone or in coalition with 246 other angiogenic factors such as PlGF. This ensues for the requirement of more 
247 prospective studies in assessing the role of sTWEAK alone and comparing its 248 prognostic performance when combined with other biomarkers. Despite the limitations,

249 this is perhaps the first prospective study on the role of sTWEAK in diagnosing PE and 250 these findings may open ways for future researchers in assessing the molecular events 251 that lead to the disease pathogenesis.

\section{Conclusion:}

253 sTWEAK levels at early pregnancy weeks were found to be low in high risk females 254 who developed pre-eclampsia at follow up versus normotensive pregnant women. 255 Baseline TWEAK might serve as an independent variable for prediction of pre256 eclampsia; however longitudinal studies with larger sample size are required to 257 ascertain the causal relation.

258 Conflicts of interest: none declared.

259 Acknowledgment:

260 The authors wish to thank the study volunteers for their kind support and facilitation in 261 providing timely data. 
Included $(\mathrm{n}=80)$ :

$>$ High risk pregnant women (HR)

$>$ Healthy pregnant women as controls

$>$ Between 13-20 weeks of gestation

Excluded $(\mathrm{n}=11)$ :

> Chronic systemic diseases (Immunological, endocrinological and renal disease) $n=06$

$>$ Previous history of complication of pregnancy (abortion, antenatal bleeding) $n=05$

$>$ Failed to consent or lost to follow up

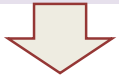

Ethical permission by concerned Institutes Informed Written Consent

\section{High Risk Group}

High risk pregnant women

$$
\mathrm{n}=63
$$

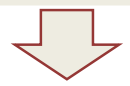

\section{Control Group}

Healthy pregnant women $\mathrm{n}=17$

Baseline (12-16weeks gestation) and follow-up (28-36 weeks gestation) Blood and Urine Collection for biomarker testing

$>$ Blood Pressure Measurements

\section{Outcome of High Risk group}

$$
\text { PIH n=24 }
$$

Normotensive $\mathrm{n}=15$

Gestational Diabetes $n=2$ 


\begin{tabular}{|c|c|c|c|c|}
\hline & & $\begin{array}{c}\text { High risk group } \\
n=63\end{array}$ & $\begin{array}{c}\begin{array}{c}\text { Negative Control } \\
\mathbf{n}=\mathbf{1 7}\end{array} \\
\end{array}$ & $P$ value \\
\hline \multicolumn{2}{|c|}{ Age (year) } & $27.05 \pm 5.89$ & $30.65 \pm 9.40$ & 0.474 \\
\hline \multicolumn{2}{|c|}{ Weight (kg) } & $68.65 \pm 19.34$ & $62.71 \pm 14.22$ & 0.488 \\
\hline \multicolumn{2}{|c|}{ Body mass index $\left(\mathrm{kg} / \mathrm{m}^{2}\right)$} & $27.01 \pm 7.02$ & $24.14 \pm 5.30$ & 0.248 \\
\hline \multicolumn{2}{|c|}{ Hemoglobin (g \%) } & $11.2 \pm 1.20$ & $11.35 \pm 1.01$ & 0.334 \\
\hline \multicolumn{2}{|c|}{ Random Blood Glucose (mg/dl) } & $96.24 \pm 23.66$ & $98.45 \pm 10.27$ & 0.840 \\
\hline \multicolumn{2}{|c|}{ SGPT (U/L) } & $21.73 \pm 10.7$ & $10.00 \pm 1.22$ & 0.040 \\
\hline \multicolumn{2}{|c|}{ Alkaline Phosphatase (U/L) } & $243.3 \pm 78.36$ & $167.0 \pm 120.22$ & 0.039 \\
\hline \multicolumn{2}{|c|}{ Total Bilirubin (mg/dl) } & $0.571 \pm 0.21$ & $0.30 \pm 0.11$ & 0.145 \\
\hline \multicolumn{2}{|c|}{ Direct Bilirubin (mg/dl) } & $0.13 \pm 0.03$ & $0.10 \pm 0.02$ & 0.601 \\
\hline \multicolumn{2}{|c|}{ Serum Uric Acid (mg/dl) } & $3.91 \pm 1.09$ & $3.50 \pm 1.00$ & 0.540 \\
\hline \multicolumn{2}{|c|}{ Serum Urea (mg/dl) } & $16.45 \pm 5.76$ & $12.00 \pm 2.45$ & 0.260 \\
\hline \multicolumn{2}{|c|}{ Serum Creatinine (mg/dl) } & $0.60 \pm 0.12$ & $0.80 \pm 0.11$ & 0.250 \\
\hline \multirow{2}{*}{ Parity } & Primi & $17(26.98)$ & $14(82.23)$ & \multirow{2}{*}{$<0.01$} \\
\hline & Multi & $46(73.01)$ & $2(11.76)$ & \\
\hline \multicolumn{2}{|c|}{ Previous History of PE } & $17(26.98)$ & -- & $<0.001$ \\
\hline \multirow{2}{*}{ No of fetus } & Singleton & $60(95.23)$ & $17(100)$ & \multirow{2}{*}{0.621} \\
\hline & Multiple & $3(4.76)$ & -- & \\
\hline
\end{tabular}




\begin{tabular}{|c|c|c|c|c|c|}
\hline \multirow{2}{*}{$\begin{array}{c}\text { Blood Pressure } \\
\text { In Different } \\
\text { Gestational weeks }\end{array}$} & \multicolumn{3}{|c|}{ High Risk Group $(n=63)$} & \multirow{2}{*}{$\begin{array}{c}\text { Negative } \\
\text { Control } \\
(\mathbf{n}=17)\end{array}$} & \multirow[b]{2}{*}{$\begin{array}{c}\mathbf{p} \\
\text { value }\end{array}$} \\
\hline & $\begin{array}{l}\text { Developed } \\
\text { PE }(n=22)\end{array}$ & $\begin{array}{l}\text { Developed } \\
\text { PIH }(n=24)\end{array}$ & $\begin{array}{l}\text { Normotensive } \\
\quad(n=17)\end{array}$ & & \\
\hline $\begin{array}{l}\text { Systolic Baseline }(\mathrm{mmHg}) \text { at } \\
12 \text { weeks }\end{array}$ & $120.00 \pm 17.45$ & $118.10 \pm 15.84$ & $114.70 \pm 7.98$ & $110.59 \pm 13.44$ & 0.124 \\
\hline $\begin{array}{l}\text { Diastolic Baseline }(\mathrm{mmHg}) \text { at } \\
12 \text { weeks }\end{array}$ & $78.64 \pm 14.57$ & $75.48 \pm 13.98$ & $74.38 \pm 8.92$ & $72.35 \pm 9.701$ & 0.059 \\
\hline $\begin{array}{l}\text { Systolic Follow up at }(\mathrm{mmHg}) \\
28 \text { weeks }\end{array}$ & $159.50 \pm 17.00$ & $121.00 \pm 4.55$ & $112.35 \pm 9.70$ & $109.09 \pm 10.41$ & 0.007 \\
\hline $\begin{array}{l}\text { Diastolic Follow up at }(\mathrm{mmHg}) \\
28 \text { weeks }\end{array}$ & $93.81 \pm 16.57$ & $89.25 \pm 11.20$ & $75.29 \pm 7.99$ & $82.05 \pm 8.80$ & 0.412 \\
\hline $\begin{array}{l}\text { Systolic Follow up at }(\mathrm{mmHg}) \\
32 \text { weeks }\end{array}$ & $133.64 \pm 16.84$ & $\begin{array}{c}127.62 \pm \\
13.43\end{array}$ & $111.76 \pm 9.51$ & $108.22 \pm 5.89$ & 0.009 \\
\hline $\begin{array}{l}\text { Diastolic Follow up at }(\mathrm{mmHg}) \\
32 \text { weeks }\end{array}$ & $85.50 \pm 6.048$ & $85.59 \pm 9.735$ & $79.62 \pm 3.54$ & $79.55 \pm 8.45$ & 0.052 \\
\hline \multicolumn{6}{|c|}{ Urine Dipstick Analysis for Protein and Glucose } \\
\hline & \multicolumn{2}{|c|}{$\begin{array}{l}\text { High Risk Group } \\
(\mathrm{n}=63)\end{array}$} & \multicolumn{3}{|c|}{$\begin{array}{c}\text { Negative Control } \\
(\mathrm{n}=17)\end{array}$} \\
\hline \multicolumn{6}{|c|}{ Urine Protein } \\
\hline $\mathbf{0}$ & \multicolumn{2}{|c|}{$49(77.9)$} & \multicolumn{3}{|c|}{$15(88.23)$} \\
\hline $1+$ & \multicolumn{2}{|c|}{$5(7.9)$} & \multicolumn{3}{|c|}{$2(11.76)$} \\
\hline $2+$ & \multicolumn{2}{|c|}{$2(3.2)$} & \multicolumn{3}{|c|}{--} \\
\hline $3+$ & \multicolumn{2}{|c|}{$7(11.1)$} & \multicolumn{3}{|c|}{--} \\
\hline \multicolumn{6}{|c|}{ Urine Glucose } \\
\hline $\mathbf{0}$ & \multicolumn{2}{|c|}{$61(96.8)$} & \multicolumn{3}{|c|}{$17(100)$} \\
\hline 1 & \multicolumn{2}{|c|}{$2(3.2)$} & \multicolumn{2}{|r|}{--} & \\
\hline
\end{tabular}


Table 3: sTWEAK and PIGF Levels in subjects stratified based on HR group outcomes

\begin{tabular}{|c|c|c|c|c|c|}
\hline \multirow[b]{2}{*}{ Biomarkers } & \multicolumn{3}{|c|}{ High Risk Group $(n=63)$} & \multirow{2}{*}{$\begin{array}{c}\text { Negative } \\
\text { Control } \\
(n=17)\end{array}$} & \multirow[b]{2}{*}{ p-value } \\
\hline & $\begin{array}{l}\text { Developed } \\
\text { PE }(n=22)\end{array}$ & $\begin{array}{l}\text { Developed } \\
\text { PIH }(n=24)\end{array}$ & $\begin{array}{l}\text { Normotensive } \\
\qquad(\mathbf{n = 1 7})\end{array}$ & & \\
\hline sTWEAK Baseline (ng/dl) & $4.03 \pm 0.37$ & $5.80 \pm 0.56$ & $13.29 \pm 0.70$ & $15.10 \pm 0.64$ & $<0.001$ \\
\hline sTWEAK Follow up (ng/dl) & $1.93 \pm 0.23$ & $8.35 \pm 0.78$ & $10.13 \pm 1.10$ & $30.53 \pm 0.79$ & $<0.001$ \\
\hline PlGF (pg/ml) & $74.22 \pm 10.11$ & $89.38 \pm 8.38$ & $101.0 \pm 12.13$ & $109.82 \pm 7.83$ & 0.013 \\
\hline & $\begin{array}{l}\text { ean } \pm \text { SEM. C } \\
\text { Statistically sig }\end{array}$ & IS C & $\begin{array}{l}\text { as made by } \mathrm{T} \text { Tes } \\
\text { hotensives } \mathrm{p}<0.0\end{array}$ & 14 & \\
\hline
\end{tabular}




\section{Reference:}

314 1. Phipps E, Prasanna D, Brima W, Jim B. Preeclampsia: updates in pathogenesis, 315 definitions, and guidelines. Clinical Journal of the American Society of Nephrology. 2016;11(6):1102-13.

2. Guo X, Xu L, Huang J, Zhao M. Case-control Study on Serum Calcium and Magnesium Levels in Women Presenting with Preeclampsia. BMC Pregnancy Childbirth 2017;20(14):390.

319 3. Yildirim ZK, Sumnu A, Bademler N, Kilic E, Sumnu G, Karadag S, et al. Soluble TNF-Like 320 Weak Inducer of Apoptosis as a New Marker in Preeclampsia: A Pilot Clinical Study. molecules. 2016;12:15.

323 hypertension in pre-eclampsia: a lesson in integrative physiology. Acta Physiol. 2013;208:22433.

5. Roberts JM, Bodnar LM, Patrick TE, Powers RW. The Role of Obesity in Preeclampsia. Pregnancy hypertension. 2011;1(1):6.

6. Savaj S, Vaziri N. An overview of recent advances in pathogenesis and diagnosis of preeclampsia. Iranian journal of kidney diseases. 2012;6(5):334-8.

7. Miehle K, Stepan H, Fasshauer M. Leptin, adiponectin and other adipokines in gestational diabetes mellitus and pre-eclampsia. Clinical endocrinology. 2012;76(1):2-11.

8. Wu W-K, Georgiadis A, Copland DA, Liyanage S, Luhmann UF, Robbie SJ, et al. IL-4 Regulates Specific Arg-1+ Macrophage sFlt-1-Mediated Inhibition of Angiogenesis. The American journal of pathology. 2015;185(8):2324-35.

9. Wu P, van den Berg C, Alfirevic Z, O’Brien S, Röthlisberger M, Baker PN, et al. Early pregnancy biomarkers in pre-eclampsia: a systematic review and meta-analysis. International journal of molecular sciences. 2015;16(9):23035-56.

10. Anderson UD, Olsson $M$, Kristensen $K$, Åkerström B, Hansson S. Review: Biochemical markers to predict preeclampsia. Placenta. 2012;33:S42-S7.

11. Kar M. Role of biomarkers in early detection of preeclampsia. Journal of Clinical and Diagnostic Research. 2014;8(4):BE01-BE4.

12. Wright A, Guerra L, Pellegrino M, Wright D, Nicolaides KH. Maternal serum PAPP-A and free $\beta$-hCG at 12, 22 and 32 weeks' gestation in screening for pre-eclampsia. Ultrasound in Obstetrics \& Gynecology. 2016;47(6):762-7.

13. Sachan R, Patel ML, Dhiman S, Gupta P, Sachan P, Shyam R. Diagnostic and prognostic significance of serum soluble endoglin levels in preeclampsia and eclampsia. Advanced Biomedical Research. 2016;5(1):119.

14. De Falco S. The discovery of placenta growth factor and its biological activity. Experimental \& molecular medicine. 2012;44(1):1.

15. Rios DRA, Alpoim PN, Godoi LC, Perucci LO, de Sousa LP, Gomes KB, et al. Increased levels of SENG and SVCAM-1 and decreased levels of VEGF in severe preeclampsia. American journal of hypertension. 2015;29(11):1307-10.

16. Myers J, Kenny L, McCowan L, Chan E, Dekker G, Poston L, et al. Angiogenic factors combined with clinical risk factors to predict preterm pre-eclampsia in nulliparous women: a predictive test accuracy study. BJOG: An International Journal of Obstetrics \& Gynaecology. 2013;120(10):1215-23.

17. Ukah UV, Hutcheon JA, Payne B, Haslam MD, Vatish M, Ansermino JM, et al. Placental Growth Factor as a Prognostic Tool in Women With Hypertensive Disorders of Pregnancy: A Systematic Review. Hypertension. 2017;70(6):HYPERTENSIONAHA. 117.10150. 
18. Sato S, Ogura Y, Kumar A. TWEAK/Fn14 Signaling Axis Mediates Skeletal Muscle Atrophy and Metabolic Dysfunction. Frontiers in Immunology. 2014;5.

361 19. Lammens A, Baehner M, Kohnert U, Niewoehner J, Von Proff L, Schraeml $M$, et al. 362 Crystal structure of human TWEAK in complex with the Fab fragment of a neutralizing 20. Stephan D, Sbai O, Wen J, Couraud P-O, Putterman C, Khrestchatisky M, et al. TWEAK/Fn14 pathway modulates properties of a human microvascular endothelial cell model of blood brain barrier. Journal of neuroinflammation. 2013;10(1):9.

21. González-Sánchez DA, Álvarez CM, Vásquez G, Gómez-Puerta JA. Role of TWEAK/Fn14 signalling pathway in lupus nephritis and other clinical settings. Nefrología (English Edition). 2017;37(2):118-25.

22. Donohue PJ, Richards CM, Brown SA, Hanscom HN, Buschman J, Thangada S, et al. TWEAK is an endothelial cell growth and chemotactic factor that also potentiates FGF-2 and VEGF-A mitogenic activity. Arteriosclerosis, thrombosis, and vascular biology. 2003;23(4):594600.

23. Dean AG SK, Soe MM. OpenEpi: Open Source Epidemiologic Statistics for Public Health, Version. 2013 [updated 2013/04/06 Available from: http://www.openepi.com/Menu/OE Menu.htm

24. Kallela J, Jääskeläinen $T$, Kortelainen $E$, Heinonen $S$, Kajantie $E$, Kere J, et al. The diagnosis of pre-eclampsia using two revised classifications in the Finnish Pre-eclampsia Consortium (FINNPEC) cohort. BMC Pregnancy and Childbirth. 2016;16(1):221.

25. El-Asrar AMA, De Hertogh G, Siddiquei MM, Van den Eynde K, Opdenakker G. The Tumor Necrosis Factor Superfamily Members TWEAK, TNFSF15 and Fibroblast Growth FactorInducible Protein 14 Are Upregulated in Proliferative Diabetic Retinopathy. Ophthalmic Res. 2015;53(3):122-30.

26. Ruiz-Ortega M, Ortiz A, Ramos AM. Tumor necrosis factor-like weak inducer of apoptosis (TWEAK) and kidney disease. Current opinion in nephrology and hypertension. 2014;23(1):93-100.

27. Blanco-Colio LM, Martín-Ventura JL, Carrero JJ, Yilmaz MI, Moreno JA, GómezGuerrero C, et al. Vascular proteomics and the discovery process of clinical biomarkers: the case of TWEAK. Proteomics-Clinical Applications. 2011;5(5-6):281-8.

28. Simón-Muela I, Llauradó G, Chacón MR, Olona M, Näf S, Maymó-Masip E, et al. Reduced circulating levels of TWEAK are associated with gestational diabetes mellitus. European journal of clinical investigation. 2015;45(1):27-35.

29. Binder NK, Evans J, Salamonsen LA, Gardner DK, Tu'uhevaha J, Hannan NJ. Placental growth factor is secreted by the human endometrium and has potential important functions during embryo development and implantation. PloS one. 2016;11(10):e0163096.

30. Leaños-Miranda A, Campos-Galicia I, Berumen-Lechuga MG, Molina-Pérez CJ, GarcíaPaleta $\mathrm{Y}$, Isordia-Salas $\mathrm{I}$, et al. Circulating angiogenic factors and the risk of preeclampsia in systemic lupus erythematosus pregnancies. The Journal of rheumatology. 2015;42(7):jrheum. 141571.

31. Verlohren S, Herraiz I, Lapaire O, Schlembach D, Zeisler H, Calda P, et al. New Gestational Phase-Specific Cutoff Values for the Use of the Soluble fms-Like Tyrosine Kinase1/Placental Growth Factor Ratio as a Diagnostic Test for PreeclampsiaNovelty and Significance. Hypertension. 2014;63(2):346-52.

32. Anne Cathrine Staff SJB, Peter von Dadelszen, James M. Roberts, Robert N. Taylor,, Robert W. Powers DSC-J, Christopher W.G. Redman. Brief Review. Hypertension. 2013;61(5):932-42. 
407 33. Perucci L, Gomes K, Freitas L, Godoi L, Alpoim P. Soluble Endoglin. Transforming 408 Growth Factor-Beta. 2014;1(5).

409 34. Tikmani SS, Zahid N. Rate and Risk Factors of Stillbirth in Pakistan: A Systematic 410 Review. J Pediatr Child Nutr. 2016;2(3):100116.

411 35. Man J, Hutchinson J, Heazell A, Ashworth M, Jeffrey I, Sebire N. Stillbirth and 412 intrauterine fetal death: role of routine histopathological placental findings to determine 413 cause of death. Ultrasound in Obstetrics \& Gynecology. 2016;48(5):579-84.

414 36. Suhag A, Berghella V. Intrauterine growth restriction (IUGR): etiology and diagnosis. 415 Current Obstetrics and Gynecology Reports. 2013;2(2):102-11. 